Spectral measurements of runaway electrons by a scanning probe in the TEXTOR tokamak

T. Kudyakov, K. H. Finken, M. Jakubowski, M. Lehnen, Y. Xu, and O. Willi

Citation: Review of Scientific Instruments 79, 10F126 (2008);

View online: https://doi.org/10.1063/1.2953594

View Table of Contents: http://aip.scitation.org/toc/rsi/79/10

Published by the American Institute of Physics

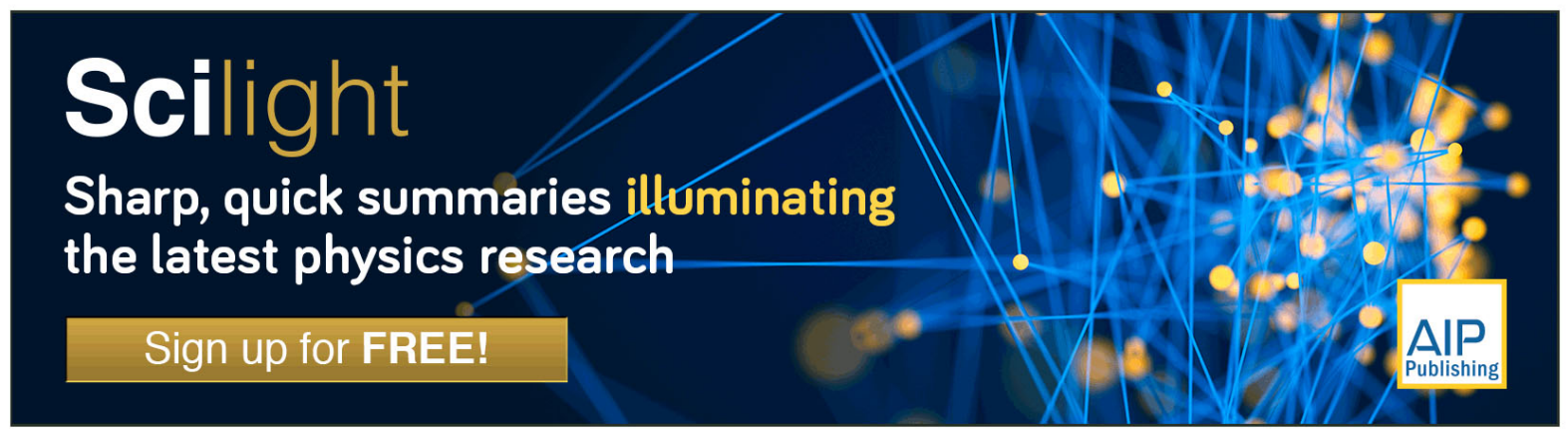




\title{
Spectral measurements of runaway electrons by a scanning probe in the TEXTOR tokamak ${ }^{\text {a) }}$
}

\author{
T. Kudyakov, ${ }^{1, b)}$ K. H. Finken, ${ }^{2}$ M. Jakubowski, ${ }^{3}$ M. Lehnen, ${ }^{2}$ Y. Xu, ${ }^{4}$ and O. Willi ${ }^{1}$ \\ ${ }^{1}$ ILPP, Heinrich-Heine-Universitaet Duesseldorf, D-40225 Duesseldorf, Germany \\ ${ }^{2}$ Institut fuer Energieforschung-Plasmaphysik, Forschungszentrum Juelich GmbH, D-52425 Juelich, Germany \\ ${ }^{3}$ Max-Planck-Institut für Plasmaphysik, IPP-EURATOM Association, D-17491 Greifswald, Germany \\ ${ }^{4}$ Laboratoire de Physique des Plasmas/Laboratorium voor Plasmafysica, ERM/KMS, EURATOM Association, \\ B-1000 Brussels, Belgium
}

(Presented 14 May 2008; received 8 May 2008; accepted 27 May 2008; published online 31 October 2008)

\begin{abstract}
Energy spectral measurements of runaway electrons were performed by a scanning probe with high spatial and temporal resolution in the TEXTOR tokamak. The probe consists of ten YSO $\left(\mathrm{Y}_{2} \mathrm{SiO}_{5}: \mathrm{Ce}\right)$ crystals, which are shielded by tungsten filters. The probe can resolve electrons with different energies between 4 and $30 \mathrm{MeV}$. An insertion of the probe to the plasma boundary several times during the discharge allowed spectral measurements of runaway electrons at different minor radial positions at the plasma edge as well as the study of runaway production in time. The high temporal resolution of the probe, $0.05 \mathrm{~ms}$, enabled measurements of runaway electrons not only during low density discharges, but also during plasma disruptions. () 2008 American Institute of Physics. [DOI: 10.1063/1.2953594]
\end{abstract}

Theoretical and experimental studies of runaway electrons have been made in most of the known tokamaks for the past few decades. The main interest in the study of superthermal electrons is mostly defined by the enormous heat flux coming to plasma facing materials with a runaway current produced during a plasma disruption. The large heat fluxes can damage the wall of large fusion machines such as JT60-U and JET and especially of ITER. ${ }^{1}$

The main processes responsible for the production of runaway electrons were introduced in Refs. 2 and 3 for the first time. The conventional or primary generation process of runaway electrons and the corresponding production rate were described in Refs. 2 and 4-7. More recently another process-avalanching or secondary generation of runaway electrons - and the related production rate were presented in Refs. 3 and $8-10$. In the secondary process, the runaway electrons are generated due to the interaction of runaway electrons with thermal electrons.

In order to study the production of runaway electrons experimentally, several different diagnostics have been developed in different tokamaks. In general all methods developed allows us to get information about runaway electrons at a specific energy range in the plasma core or at the vessel wall. At the vessel wall several techniques were applied: (i) gamma rays were observed during thick-target interactions with runaway electrons; ${ }^{11-13}$ (ii) neutrons produced in $(\gamma, n)$ photonuclear reactions due to interactions of runaway electrons with the plasma facing materials ${ }^{13,14}$ were measured; (iii) measurements of hard $\mathrm{x}$-ray radiation produced when runaway electrons impinged on the wall ${ }^{12}$ were carried out.

\footnotetext{
a) Contributed paper, published as part of the Proceedings of the 17th Topical Conference on High-Temperature Plasma Diagnostics, Albuquerque, New Mexico, May 2008.

b)Electronic mail: timur.kudyakov@uni-duesseldorf.de.
}

On the other hand, information about runaway electrons inside the plasma was obtained by synchrotron radiation in the midinfrared spectral wavelength range $(4-8 \mu \mathrm{m})$ produced by the highly relativistic electrons with energies above $25 \mathrm{MeV}$ propagating in the toroidal direction. ${ }^{15}$

In the present paper a new scanning probe implemented in the TEXTOR tokamak provided direct measurements of the electron energy spectrum between 4 and $30 \mathrm{MeV}$ at the plasma edge. The new diagnostic in addition to the synchrotron radiation and neutron techniques presents a powerful tool to study runaway electrons in the plasma volume as well as in the scrape-off layer.

The probe can be inserted $2 \mathrm{~cm}$ into the plasma for a period of less than $50 \mathrm{~ms}$. This short time is due to the heat flux of the thermal and of the runaway electrons causing damage to the probe. In the results presented the probe was inserted with a velocity of $0.7 \mathrm{~m} / \mathrm{s}$ at a minor radial position of $46 \mathrm{~cm}$, which corresponds to the position of the last closed field surface (LCFS).

The probe design and the experimental setup are shown in Fig. 1. The probe consists of ten $\mathrm{YSO}\left(\mathrm{Y}_{2} \mathrm{SiO}_{5}: \mathrm{Ce}\right)$ crystals, ${ }^{16,17}$ which are shielded by different thicknesses of tungsten filters (between 0 and $6 \mathrm{~mm}$ ) placed in the runaway electrons' direction [see Fig. 1(a)]. It is significant to note that from the other directions the crystals are properly shielded by more than $6 \mathrm{~mm}$ tungsten. The outside of the probe is covered by a $5 \mathrm{~mm}$ graphite housing. The crystals have the following characteristics: light output of 30000 photons $/ \mathrm{MeV}$, melting point of $2470{ }^{\circ} \mathrm{C}$, length of $2 \mathrm{~mm}$, cross section of $1 \mathrm{~mm}^{2}$, and decay time and peak emission of $42 \mathrm{~ns}$ and $420 \mathrm{~nm}$, respectively. The main characteristic of the crystals is that the light output does not change considerably (10\%) for $\gamma$-rays with energies higher than $100 \mathrm{keV}:{ }^{17}$ a similar result can be expected for electrons in the same energy range. A possible influence by neutrons 



FIG. 1. (a) The probe design showing the positions of the YSO crystals inside the graphite housing. The light produced in the crystals is transferred to photomultipliers by optical fibers. (b) The experimental setup.

produced in $(\gamma, n)$ photonuclear reactions, when a runaway electron hits the graphite wall of the probe or of the carbon limiter, can be neglected since the YSO is a low $Z$ material and is only sensitive to $\gamma, e^{-}$, and $e^{+}$particles. The insensitivity of the YSO crystals to neutrons was also confirmed in a test discharge when a large flux of neutrons was produced.

Light produced by the incident electrons in the crystals is transferred by optical fibres to Hamamatsu R3896 photomultiplier tubes. With this setup a temporal resolution of $0.05 \mathrm{~ms}$ and a spatial resolution of $2 \mathrm{~mm}$ were obtained. The probe was located in the equatorial midplane of TEXTOR at the low field side, as can be seen in Fig. 1(b).

The analysis of the measured energy range by the probe was carried out using the Monte Carlo GEANT4 simulation code. ${ }^{18}$ In the simulations a real three dimensional (3D) geometry of the probe and a monoenergetic electron beam source were applied. Simulations were carried out for electron beams with different energies. However, for each simulation the number and energy of the electrons were kept constant. The absorbed energy in the crystal per one electron (gamma) passing through the crystal is a slowly varying function of the electron (gamma) energy between 1 and $30 \mathrm{MeV}$. The deposited energy in the crystal is about $700 \mathrm{keV}(15 \mathrm{keV})$ per one electron (gamma). Since the absorbed energy does not strongly depend on the electron (gamma) energy, the signal produced by an electron in the crystal is two orders of magnitude higher than by a gamma with the same energy.

The total absorbed energy in 3D GEANT4 simulations by each crystal [see Fig. 2(a)] reduces proportionally with increasing thickness of the tungsten filters. The minimum electron energy registered by each crystal is defined from the approximation that the total absorbed energy in the appropriate crystal reduces ten times from its maximum value. In Fig. 2(b), the minimum measured electron energy by each crystal is shown. Therefore the probe can resolve electrons with energies between 5 and $30 \mathrm{MeV}$.

The spectral analysis of the measured relativistic electron beam has been implemented under certain conditions: (i) every crystal can register electrons with energies higher

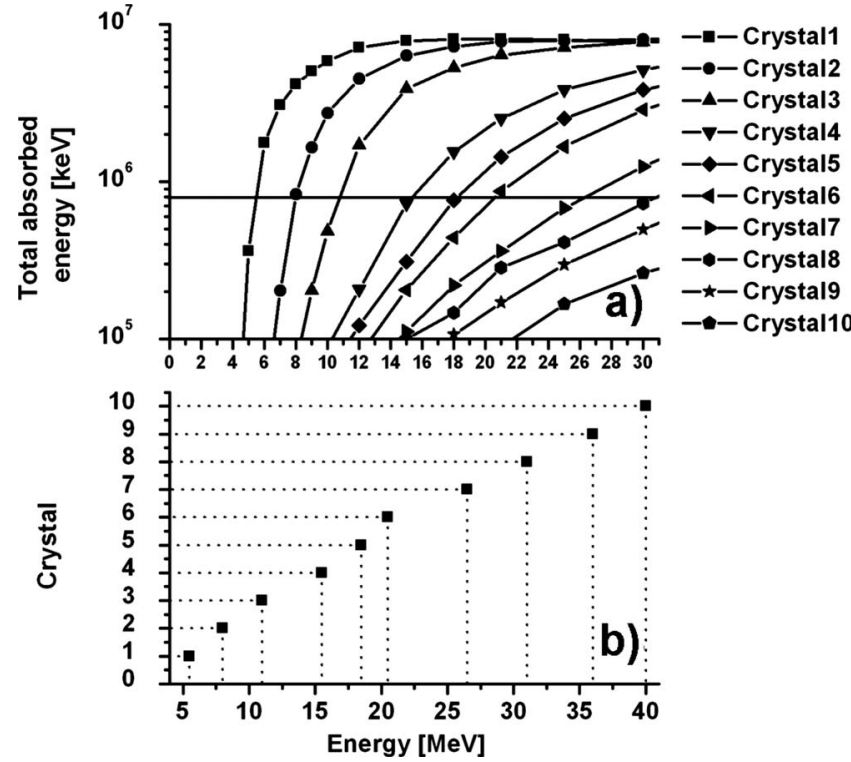

FIG. 2. (a) 3D Monte Carlo GEANT4 code simulations of the total absorbed energy by each crystal in the probe. (b) Electron energy range measured by the probe.

than the corresponding minimum energy [see Fig. 2(b)], (ii) all electrons registered by the crystal produce the same light output. The second condition is fulfilled by the characteristics of the crystal ${ }^{17}$ and due to the fact that electrons with energies in the measured energy range lost almost the same amount of energy in the crystal. The minimum detected energy has been defined under the assumption that the absorbed energy reduces by a factor of 10 . In this case the measured spectrum will not be substantially modified by the probe itself due to the absorption of part of the transported electrons in the tungsten filters. At the same time the influence of less energetic electrons on the produced signal leads to an error in the spectral measurements of about $20 \%$.

In the TEXTOR tokamak electrons can be accelerated up to a maximum energy of $30 \mathrm{MeV}$. This maximum is defined by the synchrotron radiation losses. ${ }^{15,19}$ In order to get information about the runaway electrons' spectrum in the energy range between 4 and $30 \mathrm{MeV}$, signals from the first seven crystals were used. Light intensities produced in each crystal can be written as

$$
\begin{aligned}
& I_{1}=\alpha n_{1}+\alpha n_{2}+\cdots+\alpha n_{7}, \\
& \cdots, \\
& I_{i}=\alpha n_{i}+\cdots+\alpha n_{7}, \\
& \cdots, \\
& I_{7}=\alpha n_{7},
\end{aligned}
$$

where $I_{i}$ is the light intensity produced in the $i$ th crystal, $\alpha$ is the light intensity produced by one electron, and $n_{i}$ is the number of electrons with energies between two minimum energies defined for the $i$ th and $(i+1)$ th crystals (for example, $n_{1}$ is the number of electrons with energies between 5 and $8 \mathrm{MeV}$ ). Hence the total number of electrons, $N_{e}$, with energies between 4 and $30 \mathrm{MeV}$ is $N_{e}=\sum_{i=1}^{7} n_{i}$. Consequently, the distribution of electrons in the defined energy range can 


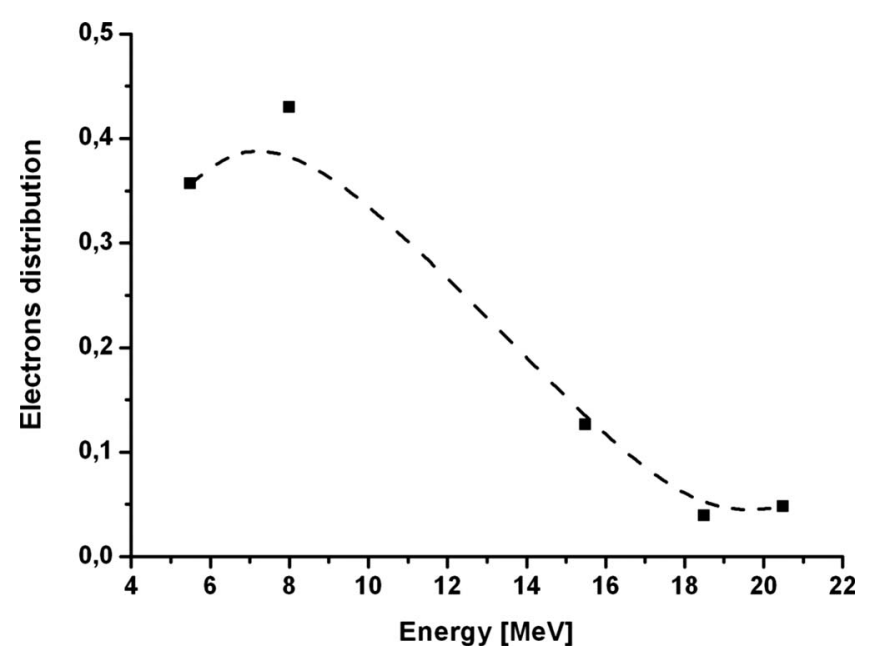

FIG. 3. Runaway electron spectrum measured during a low density plasma discharge. The probe was inserted to the LCFS at $1.15 \mathrm{~s}$ into the discharge.

be found from the ratio of the light intensities in the crystals.

Measurements of the runaway electron spectrum have been performed with the following plasma parameters: plasma density $n_{e}=0.9 \times 10^{19} \mathrm{~m}^{-3}$, plasma current $I_{p}$ $=300 \mathrm{kA}$, and magnetic field $B_{t}=2.25 \mathrm{~T}$. The probe was inserted to the LCFS at $1.15 \mathrm{~s}$ during the discharge. The measured runaway electron spectrum is shown in Fig. 3.

In conclusion, a new diagnostic has been developed to measure the spectrum of runaway electrons at the plasma edge. The high temporal resolution allows the spectrum of the superthermal electrons produced during plasma disruptions to be measured. In combination with synchrotron radiation and neutron measurements, the runaway electrons were studied in the interior and exterior regions of the plasma.
The authors acknowledge the assistance of the staff at TEXTOR and A. Hiller. Funding of this work by the virtual institute, GRK1203 F and E contract, and "Partners in the Trilateral Euregio Cluster" (TEC) is acknowledged. We acknowledge the TEXTOR team.

${ }^{1} 1999$ ITER Physics Basis, Nucl. Fusion 39, 2137 (1999).

${ }^{2}$ H. Dreicer, Phys. Rev. 115, 238 (1959).

${ }^{3}$ Yu. A. Sokolov, JETP Lett. 29, 218 (1979).

${ }^{4}$ A. V. Gurevich, Sov. Phys. JETP 12, 904 (1961).

${ }^{5}$ M. D. Kruskal and I. B. Bernstein, PPPL Report No. MATT-Q-20, 1962, p. 74 .

${ }^{6}$ R. H. Cohen, Phys. Fluids 19, 239 (1976).

${ }^{7}$ J. W. Connor and R. J. Hastie, Nucl. Fusion 15, 415 (1975).

${ }^{8}$ N. T. Besedin and I. M. Pankratov, Nucl. Fusion 26, 807 (1986).

${ }^{9}$ R. Jayakumar, H. H. Fleischmann, and S. J. Zweben, Phys. Lett. A 172, 447 (1993).

${ }^{10}$ M. N. Rosenbluth and S. V. Putvinski, Nucl. Fusion 37, 1355 (1997).

${ }^{11}$ B. Esposito, R. Martin-Solis, P. van Belle, O. N. Jarvis, F. B. Marcus, G. Sadler, R. Sanchez, B. Fischer, P. Froissard, J. M. Adams, E. Cecil, and N. Watkins, Plasma Phys. Controlled Fusion 38, 2035 (1996).

${ }^{12}$ B. Esposito, J. R. Martin-Solis, F. M. Poli, J. A. Mier, R. Sanchez, and L. Panaccione, Phys. Plasmas 10, 2350 (2003).

${ }^{13}$ B. Esposito, L. Bertalot, Yu. A. Kaschuck, D. V. Portnov, and J. R. Martin-Solis, Nucl. Instrum. Methods Phys. Res. A 476, 522 (2002).

${ }^{14}$ O. N. Jarvis, G. Sadler, and J. L. Thompson, Controlled Fusion and Plasma Heating, Proceedings of the 15th European Conference, Dubrovnik, 1988 (European Physical Society, Dubrovnik, 1988), Vol. 12B, Pt. I, p. 334.

${ }^{15}$ K. H. Finken, J. G. Watkins, D. Rusbuldt, W. J. Corbett, K. H. Dippel, D. M. Goebel, and R. A. Moyer, Nucl. Fusion 31, 859 (1990).

${ }^{16}$ YSO crystals produced by Proteus Inc. (http://www.proteus-pp.com).

${ }^{17}$ M. Balcerzyk, M. Moszyski, M. Kapusta, D. Wolski, and C. L. Melcher, IEEE Trans. Nucl. Sci. 47, 1319 (2000).

${ }^{18}$ The calculation of the minimum detected energy of electrons was done using the GEANT4 software developed by members of the Geant 4 Collaboration (http://cern. ch/geant4).

${ }^{19}$ R. Jaspers, Ph.D thesis, Eindhoven University of Technology, The Netherlands, 1995. 